\title{
How patients being treated for non-small cell lung cancer value treatment benefit despite side effects
}

\author{
Mona L. Martin ${ }^{1} \cdot$ Julia Correll $^{1}$ - Andrew Walding ${ }^{2} \cdot$ Anna Rydén $^{3}$
}

Accepted: 13 May 2021 / Published online: 31 May 2021

(c) The Author(s) 2021

\begin{abstract}
Purpose To describe symptoms and side effects experienced by patients with advanced non-small cell lung cancer (NSCLC), assess how patients allocate sensations (i.e. symptoms or side effects) to either the disease or its treatment, and evaluate how patients balance side effects with treatment benefits.

Methods Qualitative sub-studies were conducted as part of two clinical trials in patients treated for advanced NSCLC (AURA [NCT01802632]; ARCTIC [NCT02352948]).

Results Interviews were conducted with 23 patients and 19 patients in the AURA and ARCTIC sub-studies, respectively. The most commonly experienced symptoms/side effects were respiratory ( $81 \%$ of patients), digestive ( $76 \%$ ), pain and discomfort (76\%), energy-related (71\%), and sensory (62\%). Patients identified a sensation as a treatment side effect if they had not experienced it before, if there was a temporal link between the sensation and receipt of treatment, and/or if their doctors consistently told or asked them about it in relation to side effects. Themes that emerged when patients talked about their cancer treatment and its side effects related to the serious nature of their advanced disease and their treatment expectations. Patients focused on treatment benefits, wanting a better quality of life, being hopeful, not really having a choice, and not thinking about side effects.

Conclusions In these two qualitative sub-studies, patients with advanced NSCLC valued the benefits of their treatment regardless of side effects that they experienced. Patients weighed their options against the seriousness of their disease and expressed their willingness to tolerate their side effects in return for receiving continued treatment benefits.
\end{abstract}

Keywords Interview studies $\cdot$ Non-small cell lung cancer $\cdot$ Qualitative $\cdot$ Side effects $\cdot$ Symptoms $\cdot$ Treatment benefit

\section{Introduction}

Patients' perception of their treatment experience contributes important information when assessing treatment benefit [1-4]. However, patients do not always fully report the range of symptoms they experience. Under-reporting can occur on the clinicians' part too, as they process the subjective information that is provided by patients [2,3]. Multiple studies have reported that physicians and nurses underestimate symptom onset, frequency, and severity in patients in comparison to patients' ratings [5-8]. Knowing about

Anna Rydén

anna.ryden@astrazeneca.com

Health Research Associates, Inc., Seattle, WA, USA

2 AstraZeneca R\&D, Cambridge, UK

3 AstraZeneca, Gothenburg, Sweden patients' treatment expectations, previous experience with side effects, current difficulties with tolerating side effects, and views of the balance between the benefits of treatment and the need to tolerate side effects, is important both to the drug development process and to regulatory agencies when considering the overall safety of newly developed compounds. This information is particularly relevant to new cancer therapies, which have diverse mechanisms of action, heterogeneous side effect profiles, and are often administered on a daily basis over prolonged treatment periods [9].

The standardized adverse event reporting process in clinical trials does not usually include patient perceptions of symptom severity and the degree of bother or difficulty caused by various symptoms. Without understanding how patients process the experience of symptoms and impacts, which can range from not-at-all to very severe, frequent, bothersome, or difficult-to-cope-with, it is hard to know how these aspects affect patients' willingness to tolerate 
side effects in return for an actual or anticipated treatment benefit. Patient self-assessment of tolerability could potentially increase early withdrawal from clinical trials; however, it may also be a driver of more comprehensive reporting of side effects in trials, providing an enhanced understanding of the safety profile of a treatment. The US Food and Drug Administration Oncology Center of Excellence recently identified patient-focused drug development as an important program to advance cancer therapeutic development [9].

Information on symptoms and side effects was collected in two qualitative sub-studies of patients with non-small cell lung cancer (NSCLC): the AURA and the ARCTIC sub-studies. The AURA sub-study was conducted as part of the phase I/II AURA clinical trial, which assessed treatment for NSCLC with osimertinib, an epidermal growth factor receptor (EGFR) tyrosine kinase inhibitor (TKI) [3, 10]. The ARCTIC sub-study was conducted as part of the phase III ARCTIC clinical trial in patients with advanced NSCLC treated with durvalumab, a programmed cell death ligand 1 (PD-L1) antibody, and tremelimumab, an anticytotoxic T-lymphocyte-associated antigen 4 (CTLA-4) $[11,12]$.

Previous results from the AURA sub-study showed that some symptoms or side effects that were reported only by a small number of patients received high severity and bothersomeness scores, although patients rated their overall difficulty coping with side effects as low (mean difficulty with coping score: 2.0 [standard deviation: 2.3 ] out of a possible maximum of 10.0) [3]. It is not clear whether the small numbers of patients reporting the more severe and bothersome symptoms were insufficient to affect the overall difficulty with coping ratings, or whether something independent of the perceived severity and bothersome levels was driving how patients acted on their experiences of symptoms and side effects. Possibly there is something unique about how patients view side effects from cancer treatment compared with other types of treatments and other therapeutic conditions.

The current research uses data from the AURA and ARCTIC qualitative sub-studies to describe the symptoms and side effects experienced by patients with advanced NSCLC, to assess how patients allocate sensations (i.e. symptoms or side effects) to either the disease or its treatment, and to evaluate how patients balance side effects with treatment benefits. Qualitative interview data were collected in both sub-studies to provide a better understanding of the patientperceived experience. The interview questions were similar across the two studies, allowing the data from the AURA and ARCTIC sub-studies to be combined to increase the sample size and the amount of information available for exploring patient perceptions of side effects and treatment benefit.

\section{Methods}

\section{Study design and participants}

The current report presents results from the phase I/II AURA and phase III ARCTIC trial qualitative sub-studies. The qualitative analysis used in this research was based on similarity of content, where coding and theme development explicitly reflected the content of the interview (semantic type of thematic analysis) [13-15]. The theoretical framework was a qualitative content analysis and an inductive approach was used to develop concept codes.

\section{AURA}

AURA was an open-label dose-escalation and dose-expansion study of osimertinib in adult patients with locally advanced or metastatic NSCLC, who had EGFR-TKI-sensitizing mutations or who had received prior clinical benefit from EGFR-TKI treatment, and who had disease progression while receiving previous treatment with an EGFR-TKI (ClinicalTrials.gov ID: NCT01802632) [10]. The study was conducted across seven sites in the USA, UK, Spain, and South Korea. Patients who received the study drug were invited to participate in the qualitative sub-study [3].

\section{ARCTIC}

ARCTIC was a randomized, open-label study in adult patients with locally advanced or metastatic NSCLC, who did not have EGFR tyrosine kinase-activating mutations or anaplastic lymphoma kinase rearrangements, and who had received at least two prior systemic anti-cancer regimens, including one platinum-based chemotherapy (NCT02352948) [11, 12]. ARCTIC assessed the clinical activity and safety of durvalumab versus standard of care (erlotinib, gemcitabine, or vinorelbine) in patients with $\geq 25 \%$ of tumor cells (TCs) expressing PD-L1, and the combination of durvalumab and tremelimumab versus standard of care in patients with $<25 \%$ of TCs expressing PD-L1 [11]. The study was conducted in 26 countries across North America, Latin America, Asia, and Europe. Patients who were interested in participating in the sub-study were identified during the clinical trial recruitment and enrollment procedures at 14 participating sites across the USA, UK, and Canada.

\section{Interviews}

Study coordinators invited all patients being enrolled in the AURA and ARCTIC clinical trials to participate in the 
qualitative interviews. All patients who accepted participation were interviewed. Patients declined participation if they felt too ill or had competing concerns about disease progression and had other overriding concerns and needs. All patients who accepted participation were interviewed. No tracking was done regarding responder status from the clinical trial data as it did not directly relate to the purpose of the qualitative interviews.

For the current qualitative analyses, data were obtained from telephone interviews conducted approximately 4 to 6 weeks after the initiation of study treatment. Each individual interview lasted approximately $30 \mathrm{~min}$. During the interviews, patients were asked to describe a typical day before and during clinical trial participation, and how they experienced symptoms of NSCLC at these two time points. Patients were first asked to describe their symptoms (spontaneously and then probed), and were then asked to describe what the symptom feels like, the frequency and duration of the symptom, symptom severity, and how bothersome the symptom is. Follow-up interviews (after approximately 4 months of treatment), which were conducted to detect any longitudinal differences, do not form part of the current report. In each of the two studies, four different interviewers conducted the qualitative interviews. The interviewers were experienced in qualitative interview techniques and had received training on how to use the interview guides through a process of mock interview sessions.

Semi-structured interview guides were used to focus patients on the symptoms and side effects that they had experienced before trial entry, as well as those that they experienced during the trial. Patients were also asked to discuss their expectations of their current treatment, what they thought of the balance between the risks and the benefits of their current treatment, and any thoughts they had about how they defined treatment success. Figure 1 provides an overview of the content covered in the interview guides.

\section{Qualitative analysis}

All interviews were audio-recorded and transcribed for analysis. For non-English language interviews, recordings in the AURA sub-study were first transcribed and then translated into English, whereas in the ARCTIC sub-study interviews were simultaneously translated into English for coding. To group and organize quotations that had similar content, transcripts were coded using a coding framework and ATLAS.
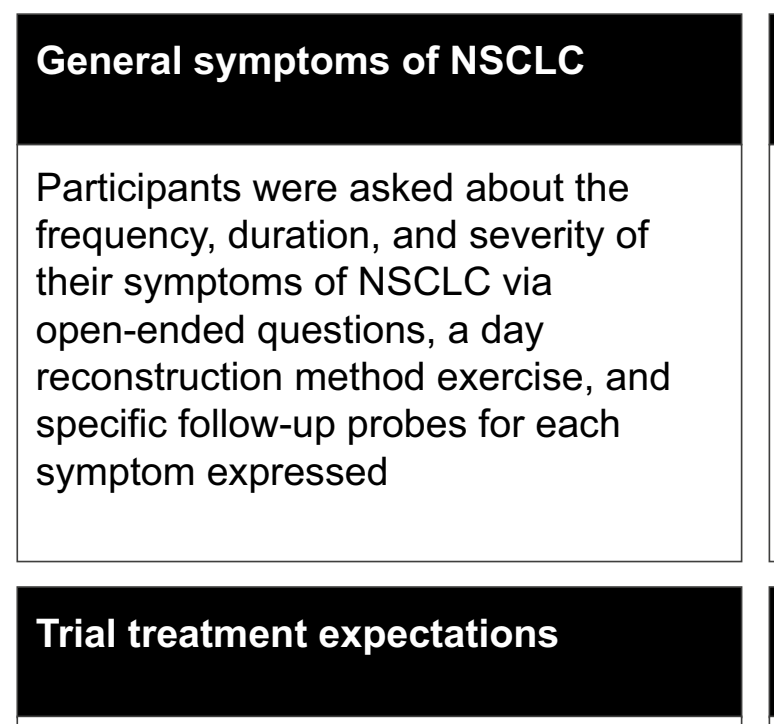

Participants were asked to describe their hopes for and expectations of the trial medication, as well as what they consider to be a successful treatment

\section{Side effects of study treatment}

Participants were asked about the side effects of the study treatment via open-ended questions and probing questions for all side effects that were not mentioned spontaneously; they were also asked about why they had attributed the side effects to the study treatment

\section{Valuation of treatment benefit and side effects}

Participants were asked whether they knew that they may experience side effects due to the study treatment, and how they balance the potential risk of side effects with the potential treatment benefit

Fig. 1 Key topics in interview guide. NSCLC non-small cell lung cancer 
ti $^{\text {TM }}$ software (versions 7.0 and 7.5 in the AURA and ARCTIC sub-studies, respectively; ATLAS.ti Scientific Software Development GmbH, Berlin, Germany).

Data quality was evaluated for data completeness, and for consistency of assigned codes. Seven transcripts were independently dual-coded and assessed for inter-coder agreement. Three coders were used for this study and consistency of coding was characterized by the overall percent of agreement in the identification of concepts, and the assignment of codes to each identified concept. Saturation of concept was evaluated by ordering the transcripts chronologically and then creating groups of five or six transcripts each. Concepts in the first transcript group were coded, and then the newly appearing codes from each subsequent group of transcripts were compared with the codes established in previous groups to assess whether any new concept codes had arisen. Saturation of concept was considered to be reached when no new concepts appeared [16].

\section{Ethics approval}

The AURA and ARCTIC trials and sub-studies were performed in accordance with the ethical principles that have their origin in the Declaration of Helsinki and were consistent with Good Clinical Practice guidelines and the applicable regulatory requirements. The trials and sub-studies were approved by the Institutional Review Boards or independent Ethics Committees (listed in full in the appendix). All data collected were handled as strictly confidential in accordance with local, state, and federal laws. The AURA and ARCTIC sub-studies posed no known risks to the patients.

\section{Results}

\section{Patients and data quality}

Interviews were conducted with 23 patients in the AURA sub-study and with 19 patients in the ARCTIC sub-study. All patients in the AURA sub-study were being treated with osimertinib. At the first interview in the ARCTIC sub-study, 10 patients were receiving treatment with durvalumab, eight with durvalumab plus tremelimumab, and one with standard of care. The median age in the AURA sub-study was 62 years (range 33-82 years), and 35\% were male [3]. In the ARCTIC sub-study, the median age was 66 years (range 44-70 years), and 58\% were male.

In the AURA sub-study, no new concepts appeared after the third of four groups of transcripts, and agreement of codes assigned ranged from $92.0 \%$ to $95.5 \%$ between coders [3]. In the ARCTIC sub-study, no new concepts appeared after the third of four groups of transcripts, and agreement on the assignment of concept codes ranged from $90.5 \%$ to $100.0 \%$ among the three coders.

\section{Predominance of concept expressions}

Table 1 shows the numbers and proportions of patient expressions and of the interview transcripts that contributed to each of the symptom or side effect concepts in the AURA and ARCTIC sub-studies. The predominance of an expression was a measure of how much it was talked about by patients compared with everything else that they mentioned. The percentage of individual patient interview transcripts that contributed a coded concept indicated how many different patients contributed to that concept.

In each of the two sub-studies, more than half of the patients talked about experiencing respiratory $(81 \%$ of patients overall), digestive $(76 \%)$, pain and discomfort (76\%), energy-related (71\%), and sensory (62\%) symptoms or side effects. There were no marked differences between the ARCTIC and the AURA sub-studies regarding the proportions of transcripts contributing to each of the concept expressions (Table 1). However, for most concept expressions, the proportion of patients contributing was larger in the ARCTIC sub-study than in the AURA substudy. The largest absolute percentage difference between the two study groups was for skin and nail-related symptoms or side effects, which $74 \%$ of patients in the AURA sub-study spoke about compared with $42 \%$ of patients in the ARCTIC sub-study. These differences reflected safety data from the two trials, which showed a relatively high incidence ( $>20 \%)$ of rash and nail-related side effects with osimertinib in the AURA study, whereas such side effects were not as commonly seen in the ARCTIC study.

\section{What were patients' expectations regarding clinical trial enrollment and successful treatment?}

When patients were asked during the qualitative interview process to describe what it was that they hoped to attain by participating in the clinical trial, the responses in the two sub-studies fell into four main themes, covering a range of desires: 'hope to maintain or regain quality of life,' 'hope that the cancer shrinks or stops growing,' 'hope for a longer life,' and 'hope for a cure.' Examples of quotations from each of the themes are shown in Table 2.

Patients were asked during the interview to describe what they would consider to be a successful treatment, in order to explore how patients thought about and recognized treatment benefits. The descriptions were broadly similar to those of the desired outcomes of trial participation and fell into three main themes: 'treatment that provides improved quality of life,' 'treatment that makes 
Table 1 Coded data frequency for symptom or side effect concepts

\begin{tabular}{|c|c|c|c|c|}
\hline \multirow[b]{2}{*}{ Symptoms or side effects } & \multicolumn{2}{|c|}{ AURA sub-study $(N=23)$} & \multicolumn{2}{|c|}{ ARCTIC sub-study $(N=19)$} \\
\hline & $\begin{array}{l}n(\%) \text { of } 662 \text { lan- } \\
\text { guage expressions }\end{array}$ & $\begin{array}{l}n(\%) \text { of } 23 \\
\text { transcripts }\end{array}$ & $\begin{array}{l}n(\%) \text { of } 359 \text { lan- } \\
\text { guage expressions }\end{array}$ & $\begin{array}{l}n(\%) \text { of } 19 \\
\text { transcripts }\end{array}$ \\
\hline Energy-related $^{\mathrm{a}}$ & $85(13)$ & $15(65)$ & $60(17)$ & $15(79)$ \\
\hline Pain and discomfort ${ }^{\mathrm{b}}$ & $105(16)$ & $15(65)$ & $67(19)$ & $17(90)$ \\
\hline Respiratory $^{c}$ & $132(20)$ & $18(78)$ & $83(23)$ & $16(84)$ \\
\hline Digestive $^{\mathrm{d}}$ & $90(14)$ & $16(70)$ & $59(16)$ & $16(84)$ \\
\hline Sleep disturbances ${ }^{\mathrm{e}}$ & $6(1)$ & $5(22)$ & $12(3)$ & $8(42)$ \\
\hline Oral $^{\mathrm{f}}$ & $33(5)$ & $9(39)$ & $8(2)$ & $6(37)$ \\
\hline Skin and nail ${ }^{\mathrm{g}}$ & 140 (21) & 17 (74) & $25(7)$ & $8(42)$ \\
\hline Sensory $^{\mathrm{h}}$ & $34(5)$ & $11(48)$ & $23(6)$ & $12(63)$ \\
\hline Genitourinary ${ }^{\mathrm{i}}$ & $1(0)$ & $1(4)$ & $0(0)$ & $1(5)$ \\
\hline Additional $^{\mathrm{j}}$ & $36(5)$ & $8(35)$ & $22(6)$ & $11(58)$ \\
\hline
\end{tabular}

${ }^{a}$ Includes exhaustion, fatigue, low energy, low stamina, tiredness, and weakness

${ }^{b}$ Includes abdominal pain, achiness, back pain, chest pain, eye pain, headache, fibromyalgia, hip-waist pain, joint or bone pain, mouth pain, muscle pain or cramping, neck pain, nipple pain, pain (unspecified), pain in extremities, side pain, shoulder pain, sore or painful skin, stiffness, and whole-body pain

${ }^{\mathrm{c} I n c l u d e s}$ bronchitis, coughing, coughing-up blood, difficulty breathing, dry nose, fluid in lungs, phlegm or mucus, pneumonia, runny nose or cold, shortness of breath, and stridor or wheezing

${ }^{\mathrm{d}}$ Includes black stool, bowel incontinence, constipation, diarrhea, difficulty swallowing, full stomach, gas, heartburn or acid reflux, heaving, nausea, poor appetite, vomiting, weight gain, and weight loss

${ }^{\mathrm{e}}$ Includes difficulty falling asleep, difficulty staying asleep, excessive sleep, nightmares, and reduced sleep quality

${ }^{\mathrm{f}}$ Includes coarse tongue, dry mouth, dry throat, hoarseness, mouth sores, sore gums, sore throat, vocal cord paralysis, and voice changes

${ }^{\mathrm{g}}$ Includes acne, burning, dry nails, dry skin, hair growth, itching, peeling, rash, sensitive skin, skin discoloration, and sores

${ }^{\mathrm{h}}$ Includes changes in taste, chills, dizziness, fainting, feels hot, fever, hot flashes, numbness or tingling, and vision difficulties

${ }^{\mathrm{i}}$ Includes frequent urinary tract infections and urinary odor

${ }^{\mathrm{j} I n c l u d e s}$ cognitive problems, confusion, difficulty concentrating, dry eyes, hyperthyroid, low blood pressure, memory loss, menstrual changes, mentally tired, night sweats, puffy eyes, shaky, swelling, and swollen lymph nodes cancer shrink or stop growing,' and 'treatment that cures cancer.' Example quotations are listed in Table 3.

\section{How do patients identify side effects of treatment?}

While exploring what patients took into consideration when asked to allocate a sensation (i.e. a symptom or side effect) to either the disease or its treatment, three main themes arose showing how patients came to their decisions: 'never experienced before,' 'timing of side effect,' and 'told by doctor it was a possibility.' Examples of patient explanations of these three themes are presented in Table 4. The first of these themes was about patients recognizing a sensation surfacing that they had not experienced before, and the second theme concentrated on identifying a temporal link between the sensation and the receipt of treatment. Generally, as treatment events became repetitive, and the experience of symptom or side effect sensations fell into similar repetition, it was not difficult for patients to allocate these sensations to the treatment. Finally, the most conclusive support for allocating a sensation to either the disease or its treatment came from the medical community itself. In this regard, patients' expressions ranged from considering what their doctors consistently asked them about when monitoring treatment tolerance and what their doctors specifically told them regarding what side effects they could expect.

A review of the transcript database showed occasional expressions of uncertainty about whether a sensation experienced should be allocated to the cancer or to its treatment. In these instances, the surrounding text in the transcript provided further context for the uncertainty, describing multiple treatments happening or "so much going on" in general. Some patients had previous experience with a particular symptom and were not sure whether it was continuing as a part of cancer progression or whether it was also related to the treatment. For some symptoms, patients were uncertain whether these were 


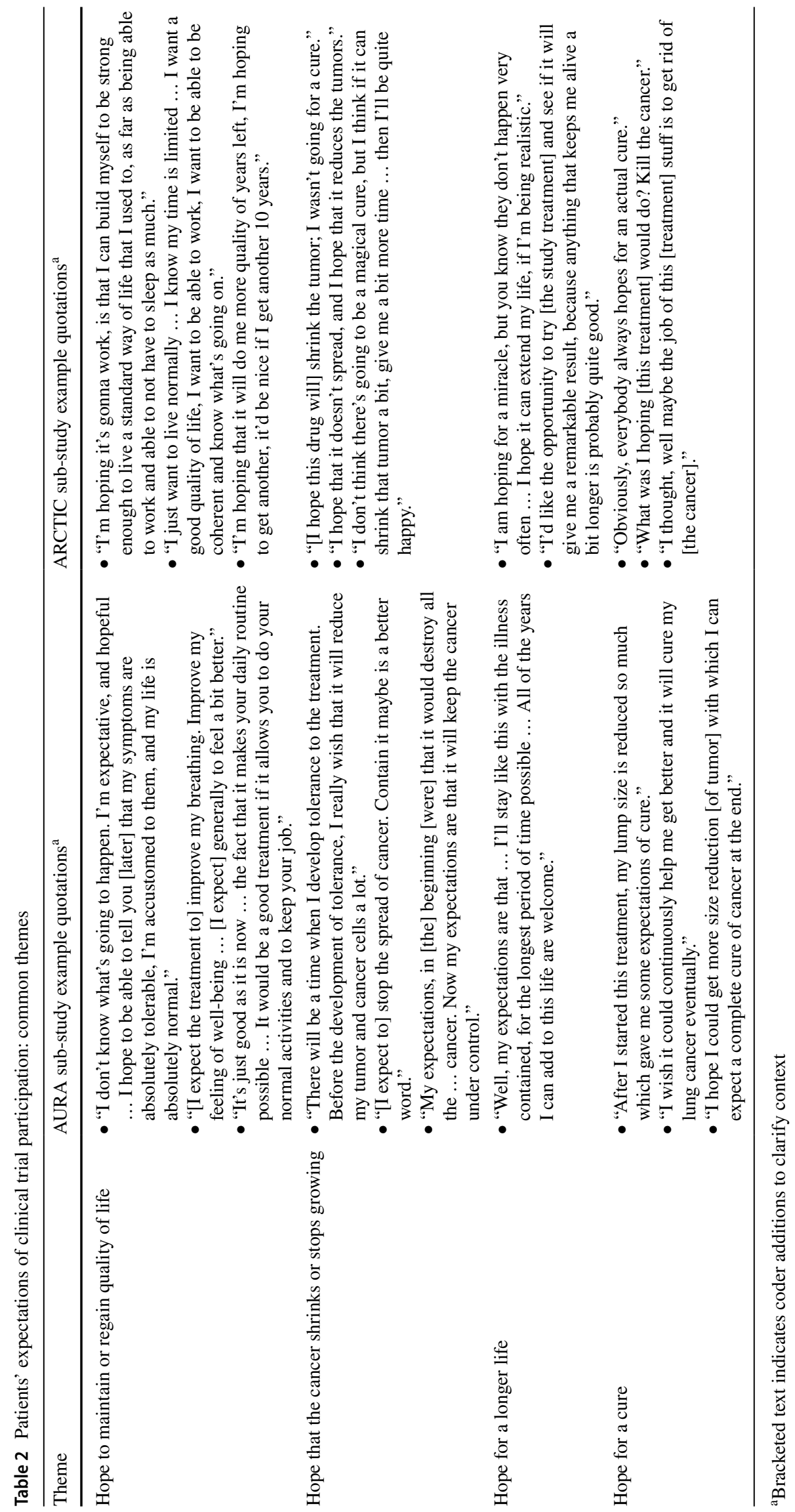




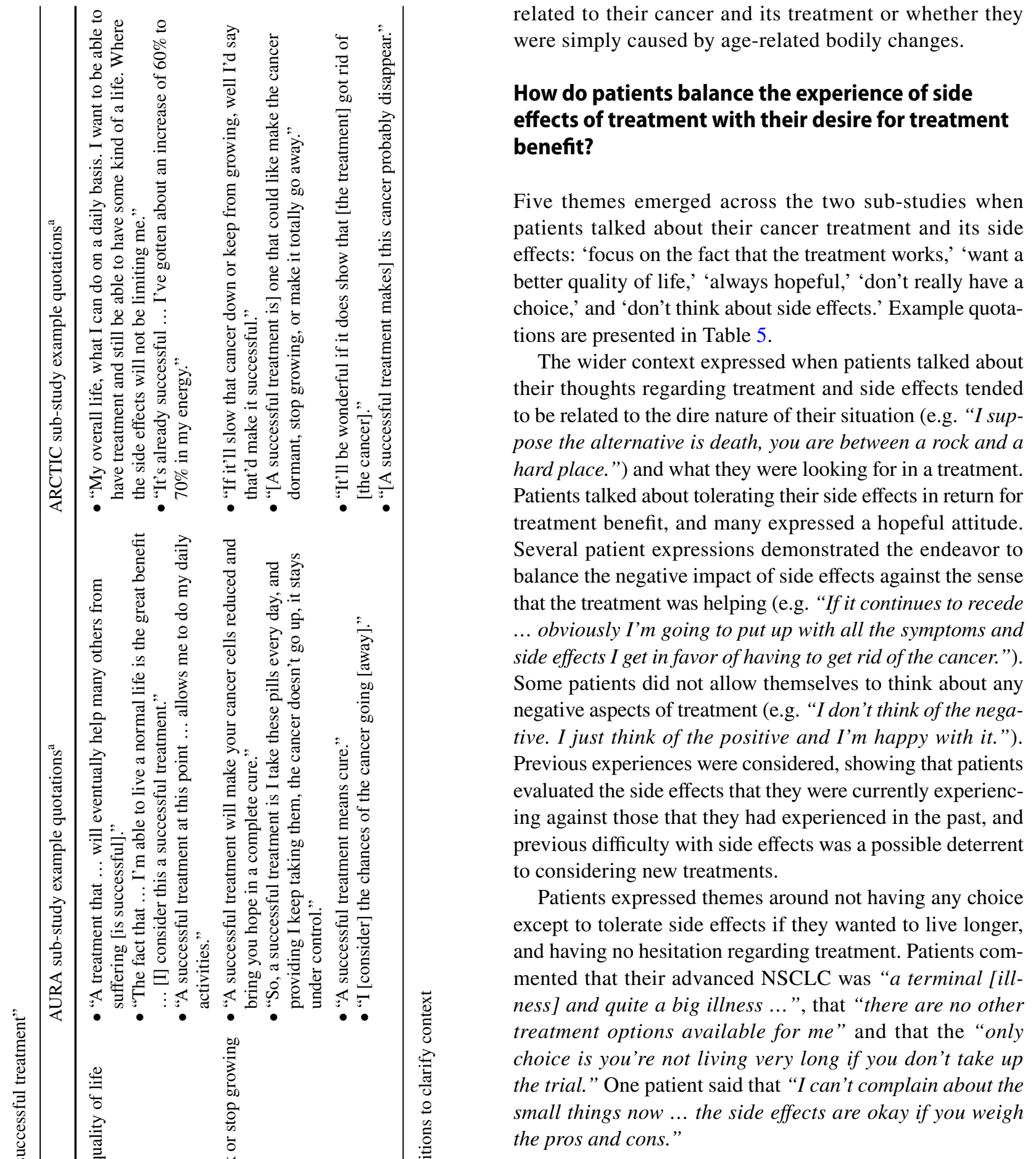

ปั

\section{Discussion}

The current article reports results from two qualitative substudies conducted as part of the phase I/II AURA and the phase III ARCTIC clinical trials. In the two sub-studies, semi-structured interviews were used to elicit qualitative data on symptoms, side effects, and expectations from patients receiving treatment for NSCLC in one of two 


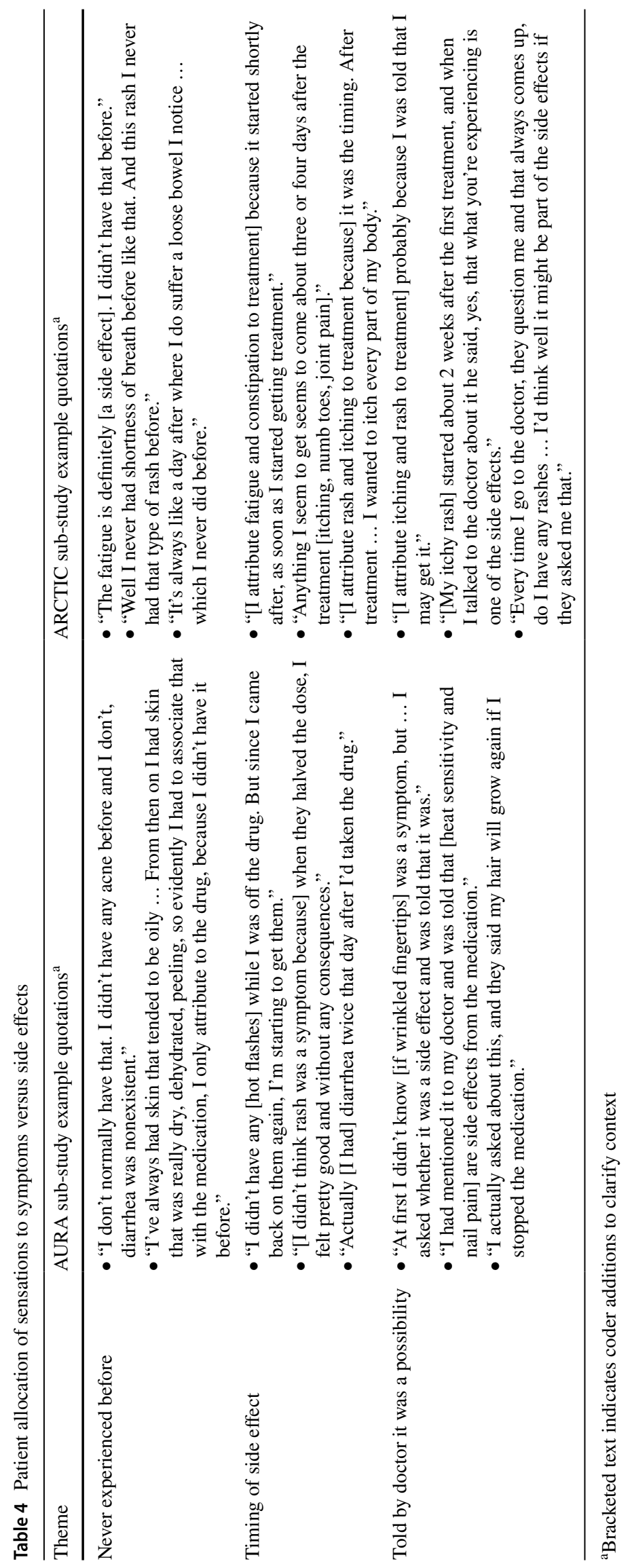




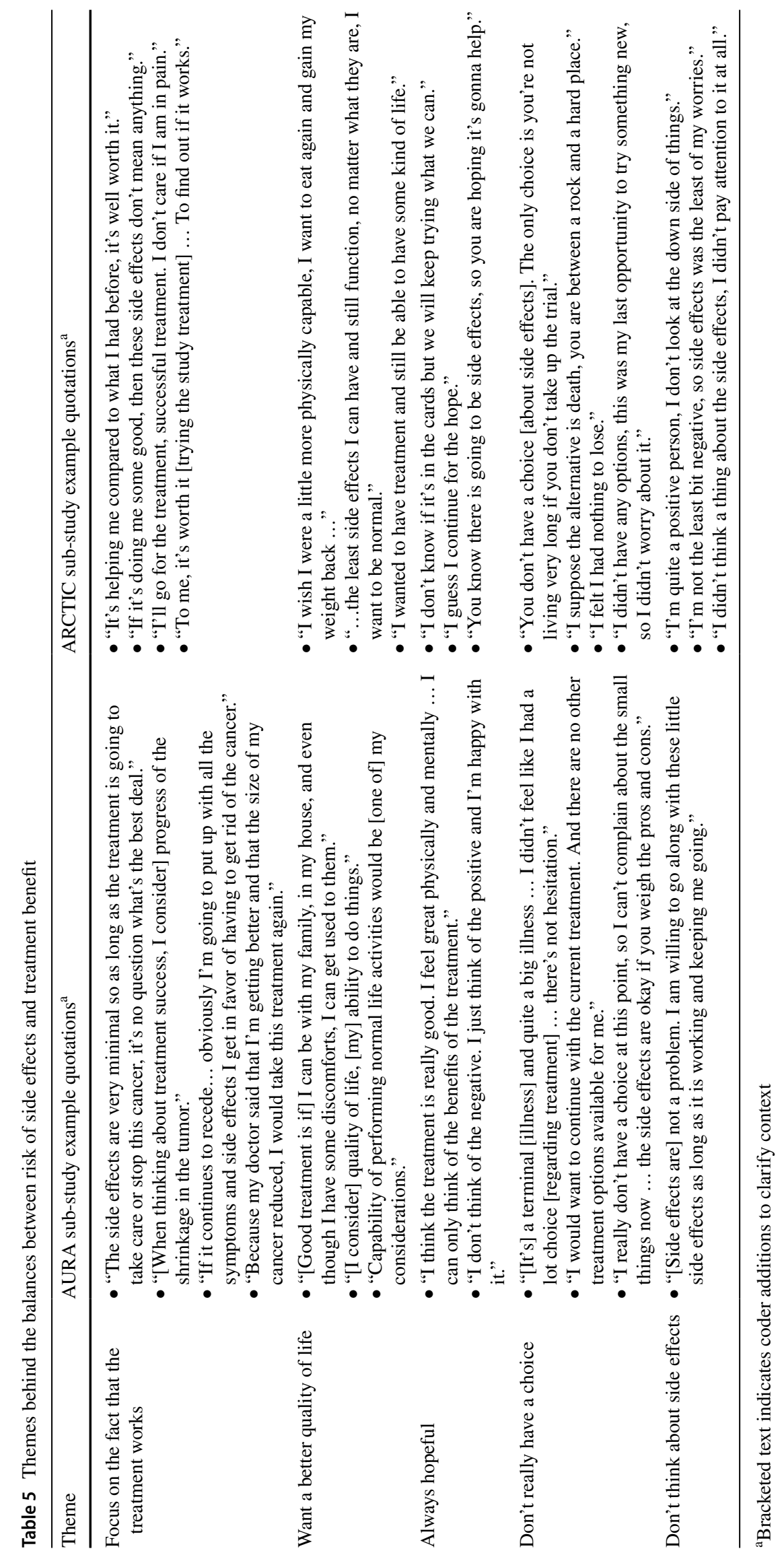


clinical trials. The aims were to describe the symptom or side effect sensations experienced by patients with NSCLC and to explore patients' expectations about clinical trial participation, and how patients balance treatment benefits and side effects.

Patients participating in the AURA sub-study or the ARCTIC sub-study were interviewed approximately 4 to 6 weeks after the initiation of their study treatment. This time frame allowed patients to become used to their new treatment and to notice any changes in the pattern of their symptom or side effect sensations. The trials both enrolled patients with advanced NSCLC, but they assessed different targeted therapies. In the AURA sub-study, patients received oral anti-cancer therapy with the EGFR-TKI osimertinib after having experienced progression of their cancer during previous EGFR-TKI therapy [10]. Patients in the ARCTIC sub-study received intravenous anti-cancer therapy with durvalumab, durvalumab plus tremelimumab, or standard of care (with erlotinib, gemcitabine, or vinorelbine) after having received at least two previous systemic treatments for their cancer, including chemotherapy $[11,12]$. Thus, patients in the ARCTIC sub-study had received more prior lines of treatment than patients in the AURA sub-study. The patterns of symptom or side effect concepts mentioned by patients in the AURA and in the ARCTIC sub-studies were generally similar, with most patients in both sub-studies talking about respiratory, digestive, and energy-related concepts, as well as pain and discomfort. A higher proportion of patients in the AURA sub-study than in the ARCTIC sub-study spoke about nail-related issues, which is in line with the known profile of osimertinib, which was the treatment assessed in the AURA trial.

Demonstrated improved quality of life and reduced toxicity are particularly important when assessing the evidence of clinical benefit from new oncology treatments in the non-curative setting [17]. Themes that arose from the substudies showed that patients were hopeful that their anticancer treatment would work, and that they were willing to tolerate treatment side effects in return for treatment benefits and a better quality of life. Not focusing on the side effects and not really having a choice but to tolerate side effects were also themes. The current results suggest that the dire nature of their cancer and the hope of a treatment benefit drove how patients acted on symptom and side effect experiences, including whether or not they reported them. Previous results from the AURA sub-study showed that, although some symptoms or side effects rated by only a few patients were scored as highly severe and highly bothersome, overall patients' difficulty coping with side effects was rated as low [3]. It appears from the current results of the two sub-studies that patients with advanced cancer cope with the perceived severity and bothersome levels of their experiences by placing them into a wider context (e.g. "If it continues to recede
... obviously I'm going to put up with all the symptoms and side effects I get in favor of having to get rid of the cancer."). It may be that patients are more willing to cope with the side effects of anti-cancer treatments than with those relating to treatment for other, more benign conditions.

Separating symptoms of a condition from the side effects of a treatment is a difficult task to ask of patients [18], and can be particularly challenging in oncology because sensations can relate to both. While it is generally believed that patients cannot correctly attribute the sensations that they experience to the disease versus its treatment because it is thought that patients do not understand the underlying clinical aspects, pathology, or pharmacology, themes that arose from the current sub-studies suggest that patients were fairly capable of distinguishing between the symptoms of their disease and the side effects of treatment. Past experience and education received from their medical team provided an important reference when patients made decisions about symptom or side effect attribution. Patients assessed whether the sensation was a new experience, in which case there was an increased likelihood that it was a treatment-related sensation. Although it is possible that new symptoms arise that are linked to the progression of cancer, this differentiation on the part of the patient presents a good foundation for sorting where the symptoms belong, particularly when combined with a temporal link between receiving a treatment and experiencing a sensation. Sensations that had been mentioned by the treating physician as potential side effects of treatment were remembered by patients, and attributed that way, which underscores the importance of physicians communicating information on potential side effects prior to the start of treatment. Results from this study demonstrate how additional information could be elicited from patients in treatment trials regarding their side effects; for example, by asking specifically about whether any new sensations have arisen since the start of the new treatment. Clinical trials may also benefit from collecting and presenting symptom or side effect information longitudinally over time from treatment initiation, to show any change in patterns over the course of the treatment and follow-up periods.

In the current sub-studies, patients' expectations regarding their clinical trial enrollment were similar in content to their descriptions of what they thought the characteristics of a successful treatment would be. Patients spoke about wanting to maintain, regain, or improve their quality of life and wanting the cancer to shrink or to stop growing. These expectations are reflected in the results of another qualitative study of patients considering participation in early phase oncology clinical trials, the large majority of whom were motivated by potential clinical benefit and about half of whom expected tumor shrinkage [19].

Saturation results suggest that continued interviews would not be likely to provide any further new information, 
and the interview results obtained were sufficient to elicit a full picture of the concepts important to this patient population. The sample size in each of the two sub-studies (23 and 19 patients in the AURA and the ARCTIC sub-studies, respectively) was typical for qualitative interview studies $[18,20,21]$. Combining the data from the AURA and ARCTIC sub-studies increased sample size and information available for exploring and comparing patient perceptions about the balance between tolerating treatment side effects and the desire for a treatment benefit. Further, the assessment of inter-rater agreement was used to evaluate the consistency of the assignment of codes to assure the different coders were processing the interview data in the same way. A total of seven transcripts were dual-coded and compared for agreement between coders in the assignment of codes to transcript text. Ideally, there should be over $90 \%$ agreement [15]. The results of this evaluation ranged from $90.5 \%$ to $100.0 \%$ for the different pairs of coders that were used.

A potential limitation of these sub-studies is that interview participants were enrolled in a clinical trial and thus may have had less comorbidity (or slightly different mixes of comorbidity) than patients treated for advanced NSCLC in the real-world patient care settings. The AURA and ARCTIC trials both excluded patients with severe or uncontrolled systemic diseases, including active bleeding diatheses or active infection. In addition, all interviewed patients had metastatic disease, and most were receiving treatment with either osimertinib or durvalumab. Also, in reference to some of the suggested consolidated criteria for reporting qualitative research (COREQ): being part of a clinical trial and under strict study protocols regarding assessments and timings, patients were not re-interviewed to gain their reflections on the thematic results of the first interviews. Finally, while the quotations provided in this report are generally from separate individuals, there are some cases where a single participant might have contributed more than one statement to the sample quotations provided. Being sourced from two different sub-studies makes it difficult to identify by individual ID numbers those cases for this manuscript. These aspects should be taken into account when considering the generalizability of this research to the broader patient population with NSCLC.

\section{Conclusions}

Qualitative results from the AURA and the ARCTIC substudies showed generally similar patterns of symptoms and side effects experienced by patients. Most patients described having pain and discomfort, as well as respiratory, digestive, and energy-related difficulties. These two sub-studies revealed a number of different influences that can affect how patients perceive and tolerate cancer treatment and its side effects. Patients identified a sensation as a treatment side effect if it had not been previously experienced, if it was temporally linked to the receipt of treatment, and/or if it had been specifically asked about or mentioned as a known side effect by the treating physician. While some patients preferred as their top priority treatments that would allow them to maintain aspects of quality of life and function, most patients expressed themes around the serious nature of having advanced NSCLC and about being willing to tolerate the negative impact of side effects to obtain treatment benefit.

Supplementary Information The online version contains supplementary material available at https://doi.org/10.1007/s11136-021-02882-6.

Funding The AURA and ARCTIC studies and sub-studies were funded by AstraZeneca. Medical writing support was provided by Anja Becher, $\mathrm{PhD}$, of Oxford PharmaGenesis, Oxford, UK, and was funded by AstraZeneca.

Data availability The data supporting the findings are available within the article.

\section{Declarations}

Conflict of interest Anna Rydén and Andrew Walding are employees of AstraZeneca and hold shares in AstraZeneca. Julia Correll and Mona L. Martin were employed for this work by Health Research Associates, which received funds from AstraZeneca to conduct the study.

Ethical approval The AURA and ARCTIC trials and sub-studies were performed in accordance with the ethical principles that have their origin in the Declaration of Helsinki and were consistent with Good Clinical Practice guidelines and the applicable regulatory requirements. The trials and sub-studies were approved by the Institutional Review Boards or independent Ethics Committees (listed in full in the appendix). All data collected were handled as strictly confidential in accordance with local, state, and federal laws. The AURA and ARCTIC sub-studies posed no known risks to the patients.

Consent to participate All participants provided their informed consent to be interviewed.

Consent to publish Not applicable.

Open Access This article is licensed under a Creative Commons Attribution 4.0 International License, which permits use, sharing, adaptation, distribution and reproduction in any medium or format, as long as you give appropriate credit to the original author(s) and the source, provide a link to the Creative Commons licence, and indicate if changes were made. The images or other third party material in this article are included in the article's Creative Commons licence, unless indicated otherwise in a credit line to the material. If material is not included in the article's Creative Commons licence and your intended use is not permitted by statutory regulation or exceeds the permitted use, you will need to obtain permission directly from the copyright holder. To view a copy of this licence, visit http://creativecommons.org/licenses/by/4.0/. 


\section{References}

1. Basch, E. (2010). The missing voice of patients in drug-safety reporting. New England Journal of Medicine, 362(10), 865-869.

2. Basch, E. (2013). Toward patient-centered drug development in oncology. New England Journal of Medicine, 369(5), 397-400.

3. Rydén, A., Blackhall, F., Kim, H. R., Pillai, R. N., Braam, L., Martin, M. L., \& Walding, A. (2017). Patient experience of symptoms and side effects when treated with osimertinib for advanced nonsmall-cell lung cancer: A qualitative interview substudy. Patient, 10(5), 593-603.

4. European Medicines Agency. (2016). Appendix 2 to the guideline on the evaluation of anticancer medicinal products in man. The use of patient-reported outcome (PRO) measures in oncology studies. https://www.ema.europa.eu/documents/other/appendix-2guideline-evaluation-anticancer-medicinal-products-man_en.pdf. Accessed 2 April 2020.

5. Fromme, E. K., Eilers, K. M., Mori, M., Hsieh, Y. C., \& Beer, T. M. (2004). How accurate is clinician reporting of chemotherapy adverse effects? A comparison with patient-reported symptoms from the Quality-of-Life Questionnaire C30. Journal of Clinical Oncology, 22(17), 3485-3490.

6. Sprangers, M. A., \& Aaronson, N. K. (1992). The role of health care providers and significant others in evaluating the quality of life of patients with chronic disease: A review. Journal of Clinical Epidemiology, 45(7), 743-760.

7. Litwin, M. S., Lubeck, D. P., Henning, J. M., \& Carroll, P. R. (1998). Differences in urologist and patient assessments of health related quality of life in men with prostate cancer: Results of the CaPSURE database. Journal of Urology, 159(6), 1988-1992.

8. Basch, E., Jia, X., Heller, G., Barz, A., Sit, L., Fruscione, M., Appawu, M., Lasonos, A., Atkinson, T., Goldfarb, S., Culkin, A., Kris, M. G., \& Schrag, D. (2009). Adverse symptom event reporting by patients vs clinicians: Relationships with clinical outcomes. Journal of the National Cancer Institute, 101(23), 1624-1632.

9. Kluetz, P. G., Kanapuru, B., Lemery, S., Johnson, L. L., Fiero, M. H., Arscott, K., Barbachano, Y., Basch, E., Campbell, M., Cappelleri, J. C., Cella, D., Cleeland, C., Coens, C., Daniels, S., Denlinger, C. S., Fairclough, D. L., Hillard, J. R., Minasian, L., Mitchell, S. A., ... Coons, S. J. (2018). Informing the tolerability of cancer treatments using patient-reported outcome measures: Summary of an FDA and Critical Path Institute workshop. Value in Health, 21(6), 742-747.

10. Jänne, P. A., Yang, J. C., Kim, D. W., Planchard, D., Ohe, Y., Ramalingam, S. S., Ahn, M.-J., Kim, S.-W., Su, W.-C., Horn, L., Haggstrom, D., Felip, E., Kim, J.-H., Frewer, P., Cantarini, M., Brown, K. H., Dickinson, P. A., Ghiorghiu, S., \& Ranson, M. (2015). AZD9291 in EGFR inhibitor-resistant non-small-cell lung cancer. New England Journal of Medicine, 372(18), 1689-1699.

11. Planchard, D., Yokoi, T., McCleod, M. J., Fischer, J. R., Kim, Y. C., Ballas, M., Shi, K., \& Soria, J. C. (2016). A phase III study of durvalumab (MEDI4736) with or without tremelimumab for previously treated patients with advanced NSCLC: Rationale and protocol design of the ARCTIC study. Clinical Lung Cancer, 17(3), 232-236.

12. Planchard, D., Reinmuth, N., Orlov, S., Fischer, J. R., Sugawara, S., Mandziuk, S., Marquez-Medina, D., Novello, S., Takeda, Y., Soo, R., \& Park, K. (2020). ARCTIC: Durvalumab with or without tremelimumab as third-line or later treatment of metastatic non-small-cell lung cancer. Annals of Oncology, 31(5), 609-618.

13. Braun, V., \& Clarke, V. Thematic analysis: A reflexive approach. https://www.psych.auckland.ac.nz/en/about/thematic-analysis. html. Accessed 5 March 2021.

14. Braun, V., \& Clarke, V. (2006). Using thematic analysis in psychology. Qualitative Research in Psychology, 3(2), 77-101.

15. Patrick, D. L., Burke, L. B., Gwaltney, C. J., Leidy, N. K., Martin, M. L., Molsen, E., \& Ring, L. (2011). Content validity - Establishing and reporting the evidence in newly developed patientreported outcomes (PRO) instruments for medical product evaluation: ISPOR PRO good research practices task force report: Part 1 - Eliciting concepts for a new PRO instrument. Value in Health, 14(8), 967-977.

16. Saunders, B., Sim, J., Kingstone, T., Baker, S., Waterfield, J., Bartlam, B., Burroughs, H., \& Jinks, C. (2018). Saturation in qualitative research: Exploring its conceptualization and operationalization. Quality \& Quantity, 52(4), 1893-1907.

17. Cherny, N. I., Sullivan, R., Dafni, U., Kerst, J. M., Sobrero, A., Zielinski, C., de Vries, E. G. E., \& Piccart, M. J. (2015). A standardised, generic, validated approach to stratify the magnitude of clinical benefit that can be anticipated from anti-cancer therapies: The European Society for Medical Oncology Magnitude of Clinical Benefit Scale (ESMO-MCBS). Annals of Oncology, 26(8), $1547-1573$.

18. Niklasson, A., Paty, J., \& Ryden, A. (2017). Talking about breast cancer: Which symptoms and treatment side effects are important to patients with advanced disease? Patient, 10(6), 719-727.

19. Dolly, S. O., Kalaitzaki, E., Puglisi, M., Stimpson, S., Hanwell, J., Fandos, S. S., Stapleton, S., Ansari, T., Peckitt, C., Kaye, S., Lopez, J., Yap, T. A., van der Graaf, W., de Bono, J., \& Banerji, U. (2016). A study of motivations and expectations of patients seen in phase 1 oncology clinics. Cancer, 122(22), 3501-3508.

20. Harrop, E., Noble, S., Edwards, M., Sivell, S., Moore, B., \& Nelson, A. (2017). Managing, making sense of and finding meaning in advanced illness: A qualitative exploration of the coping and wellbeing experiences of patients with lung cancer. Sociology of Health \& Illness, 39(8), 1448-1464.

21. Aumann, I., Kreis, K., Damm, K., Golpon, H., Welte, T., \& Matthias Graf von der Schulenburg, J. (2016). Treatment-related experiences and preferences of patients with lung cancer: A qualitative analysis. Health Expectations, 19(6), 1226-1236.

Publisher's Note Springer Nature remains neutral with regard to jurisdictional claims in published maps and institutional affiliations. 